\title{
Sistemas de protección individual anticaídas sometidos a impacto. Simulaciones numéricas
}

\section{Personal fall arrest systems under impact. Numerical simulations}

\author{
E. A. Carrión ${ }^{(*)}, \underline{\text { R. Irles }}^{(*)}$, E. G. Segovia ${ }^{(*)}$, J. C. Pomares ${ }^{(*)}$
}

\section{RESUMEN}

Los sistemas anticaída se establecen en UNE-EN-363 para evitar o retener caídas de personas sin que las fuerzas de retención causen daños relevantes. Constituyen el último recurso para prevención de caídas tras considerar otras medidas. Este artículo analiza las variables que afectan al comportamiento del sistema: altura de caída, longitud de cuerda de retención, rigidez y amortiguamiento del material de retención, y peso del operario. A tal fin, el fenómeno ha sido simulado con modelos dinámicos de elementos finitos mediante una cuerda elástica y un lastre rígido. Ello permite obtener valores verosímiles de las máximas fuerzas sobre el accidentado y el sistema durante el impacto. Los resultados demuestran el papel fundamental de la proporción entre altura de caída y longitud de cuerda (factor de caída), más determinante que la propia altura de caída. Las elevadas fuerzas máximas obtenidas indican la necesidad de incluir en el modelo absorbedores de energía elastoplásticos, en futuras investigaciones, con el fin de disminuir el impacto a valores razonables.

Palabras clave: Seguridad; protección individual; sistema anticaídas; arnés; impacto; simulación numérica.

\begin{abstract}
Personal fall protection systems are set up by EN-363 to avoid or retain people falls safely (without relevant damages). They are the ultimate resource to prevent a fall after other measures being considered. This paper analyses the variables affecting the system behaviour: free fall height, length of retaining rope, stiffness and damping of retaining material, and worker weight. To this end, fall phenomenon has been simulated with finite elements dynamic models with an elastic rope and a rigid ballast. It allows obtain likely values of maximum forces on the injured and over the system during the impact. Results demonstrate the fundamental role of height fall vs. rope length rate (fall factor), which results more important than the fall height itself. The severe values obtained for maximum forces show that future research would include elastoplastic energy absorbers to decrease impact until reasonable values.
\end{abstract}

Keywords: Safety; personal protection; fall arrest system; harness; impact; numerical simulation.

${ }^{(*)}$ Universidad de Alicante (España).

Persona de contacto/Corresponding author: ramon.irles@ua.es (R. Irles)

Cómo citar este artículo/Citation: Carrión, E. A., Irles, R., Segovia, E. .G., Pomares, J. C. (2016). Sistemas de protección individual anticaídas sometidos a impacto. Simulaciones numéricas. Informes de la Construcción, 68(542): e141, doi: http://dx.doi.org/10.3989/ic.15.050.

Licencia / License: Salvo indicación contraria, todos los contenidos de la edición electrónica de Informes de la Construcción se distribuyen bajo una licencia de uso y distribución Creative Commons Attribution License (CC BY) Spain 3.o. 


\section{INTRODUCCIÓN}

Las caídas a distinto nivel constituyen la principal causa de mortalidad por accidente de trabajo (eliminando las patologías no traumáticas, infartos y los accidentes de tráfico). Según estadísticas del Ministerio de Trabajo, promediando los datos consolidados en España de los años 2009-2012, ha habido 527.021 accidentes de trabajo anuales, de los cuales un 24,21 \% (127.613) son debidos a caídas a distinto nivel. Del total de accidentes mortales (551), los debidos a caídas ascienden a 62 , suponiendo un 11,25\% del total de los accidentes mortales.

Con el fin de evitar el riesgo o minorar las consecuencias de una caída a distinto nivel, se utiliza una gran variedad de protecciones: las protecciones colectivas (redes o barandillas) y los equipos de protección individual. Las protecciones individuales se pueden usar cuando es imposible controlar el riesgo con protecciones colectivas; por tanto, son el último recurso y como tal se debe verificar fehacientemente su efectividad.

UNE-EN 363 (1) establece cinco sistemas de protección individual contra caídas (Figura 1) en función del campo de aplicación y las solicitaciones a las que se someta en su uso' ${ }^{1}$. En este artículo se estudiará el sistema anticaídas que está sometido a impacto y como requisito básico tiene el retener la caída en condiciones de seguridad. La seguridad en la detención de la caída depende de la fuerza de parada máxima admisible, que ha sido estudiada por Sulowski (2). En la actualidad existe divergencia entre los diversos códigos de diseño (Tabla 1); en Europa este límite queda establecido en una fuerza de parada menor de $6 \mathrm{kN}$.

Los primeros estudios encontrados dedicados al análisis físico de la detención de una caída están basados en trabajos analíticos realizados por varios investigadores de manera independiente en el año 1977, Wang (3), Steinberg (4) y Microys (5), consistentes en determinar la máxima fuerza de parada (o choque) para un sistema anticaídas compuesto únicamente por un equipo de amarre y una masa rígida (lastre), obteniendo la siguiente expresión válida exclusivamente en régimen elástico proporcional.

$$
F=u \Delta_{\max }=m g\left(1+\sqrt{1+2 \frac{u h}{m g}}\right)
$$

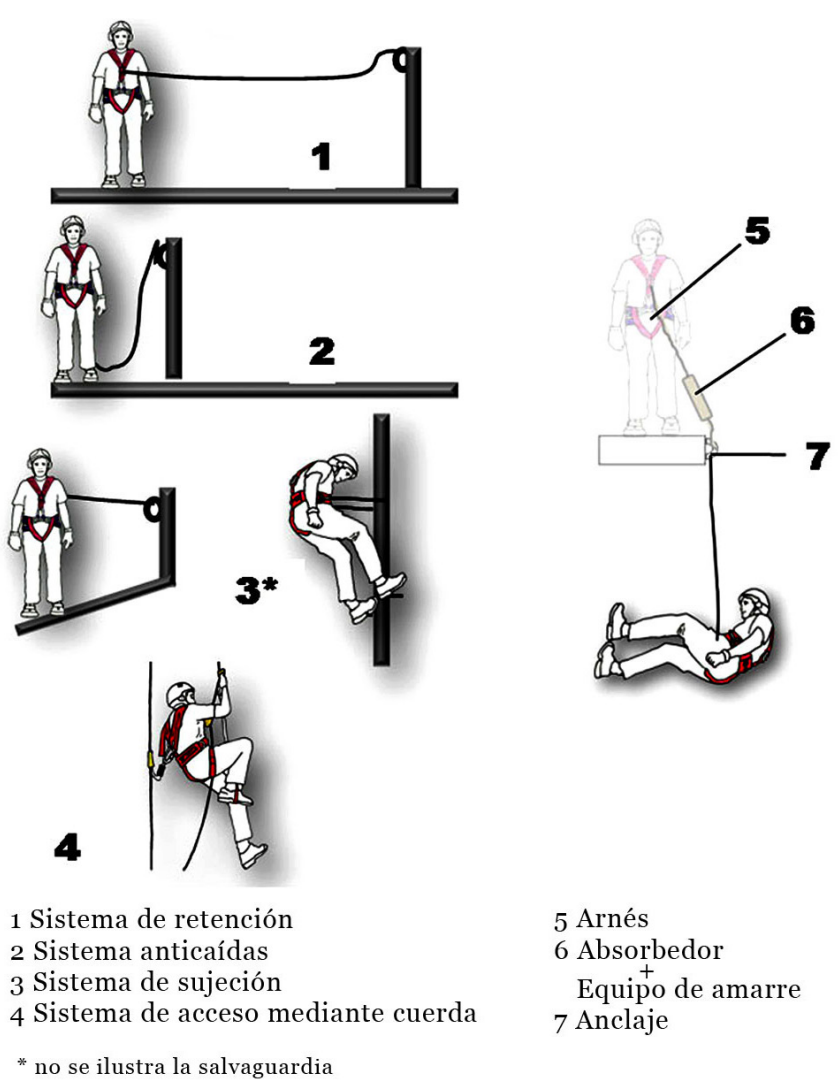

* no se ilustra la salvaguardi

Figura 1. Sistemas de protección individual contra caídas, según UNE-EN 363.

Donde:

$F$ máxima fuerza de parada

$m$ masa $(\mathrm{kg})$

$g$ aceleración de la gravedad $\left(\mathrm{m} / \mathrm{s}^{2}\right)$

$u$ gradiente de la cuerda $(u=k / L)$

$\Delta_{\max }$ elongación total máxima (estática y dinámica)

$h$ distancia de caída libre (m)

$k$ módulo de rigidez de la cuerda $(\mathrm{N})$

$L$ longitud de equipo que retiene la caída (m)

Posteriormente, en 1981, conjugando análisis empírico y analítico, la siguiente fórmula es desarrollada por Sulowski (6), contemplándose en esta expresión un sistema anticaídas con

Tabla 1. Limitaciones de Fuerza y deformación en los ensayos dinámicos en la normativa actual.

\begin{tabular}{|l|l|c|c|c|c|c|c|c|}
\hline \multicolumn{1}{|c|}{ NORMA } & TIPO & $\begin{array}{c}\text { h caída } \\
\text { (m) }\end{array}$ & $\begin{array}{c}\text { Factor } \\
\text { Caída }\end{array}$ & $\begin{array}{c}\text { N.o de } \\
\text { caídas }\end{array}$ & $\begin{array}{c}\text { Masa de } \\
\text { prueba (kg) }\end{array}$ & $\begin{array}{c}\text { Activación } \\
\text { absorbedor (kN) }\end{array}$ & $\begin{array}{c}\text { Elongación } \\
\text { (m) }\end{array}$ & $\begin{array}{c}\text { Impacto } \\
\text { máx. (kN) }\end{array}$ \\
\hline $\begin{array}{l}\text { ANSI A10.32 2004 } \\
(17)\end{array}$ & & 3,60 & 2 & 1 & 100 & 2 & 1,10 & $6,2\left(4,5^{*} 1,4\right)$ \\
\hline $\begin{array}{l}\text { ANSI Z359.1 2007 } \\
(18)\end{array}$ & & 1,80 & 1 & 1 & 100 & 2 & 1,07 & 8,0 \\
\hline $\begin{array}{l}\text { ANSI Z359.13 2009 } \\
(19)\end{array}$ & $6 \mathrm{ft} \mathrm{FF(*)}$ & 1,80 & 1 & 1 & 128 & 2 & 1,22 & 8,0 \\
\cline { 2 - 10 } & $12 \mathrm{ft} \mathrm{FF(*)}$ & 3,60 & 2 & 1 & 128 & 2 & 1,52 & 8,0 \\
\hline \multirow{2}{*}{ ISO 10333-2 (20) } & Tipo 1 & 1,80 & 1 & 1 & 100 & 2 & 1,20 & 4,0 \\
\cline { 2 - 10 } & Tipo 2 & 4,00 & 2 & 1 & 100 & 2 & 1,75 & 6,0 \\
\hline UNE-EN 355 & & 4,00 & 2 & 1 & 100 & 2 & 1,75 & 6,0 \\
\hline
\end{tabular}

(*) 6/12 feet Free Fall (caída libre de 6/12 pies).

\footnotetext{
${ }^{1}$ Se omite el sistema de salvamento.
} 
más componentes (arnés, absorbedor y dispositivo anticaídas deslizante):

$$
F=m g\left(1+\sqrt{1+\frac{2 k F_{c}}{m g}}\right) \frac{a b s}{c}
$$

Donde, además de las variables ya descritas:

$F_{c}$ factor de caída $(h / L)$

$a$ factor reductor del dispositivo anticaídas

$b$ factor reductor del dispositivo de prensión del cuerpo

$s$ factor reductor del absorbedor de energía

$c$ factor de conversión de un maniquí a un cuerpo no rígido

El factor de caída (Figura 2) que aparece en esta fórmula relaciona la altura de caída (energía cinética) con la longitud de cuerda (rigidez del sistema); más adelante se analiza el protagonismo de este factor en el fenómeno de retención de la caída.

Destacan los trabajos en el campo empírico de la biodinámica realizados en 1990 en el H.G. Amstrong Aerospace Medical Research Laboratory, Brinkley (7), en cuyos ensayos sobre voluntarios se obtuvieron datos relevantes sobre el fenómeno de la parada.

En décadas más recientes empiezan a publicarse algunos trabajos de carácter mixto (teórico-experimental) en el ámbito de la seguridad en construcción y en otros con problemas similares de retención de personas. Entre ellos, y en relación con los sistemas de protección colectiva contra caídas, pueden indicarse los trabajos de Irles (8) y Segovia (9) sobre redes de seguridad, y los de González (10) (11), Cobo (12) y Pomares (13) sobre sistemas provisionales de protección de borde.

En la actualidad los equipos de protección individual están sometidos para su comercialización en Europa a la Directiva 89/686/CEE 1407/92 (14) y a las normas armonizadas que presuponen el cumplimiento de los requisitos de seguridad y salud que establece dicha Directiva.
Los criterios de diseño y validación de los sistemas anticaídas sometidos a impacto se basan actualmente de forma exclusiva en ensayos experimentales recogidos en normas armonizadas, principalmente UNE-EN 364 y UNE-EN 355 (15) (16), realizados sobre un prototipo en laboratorio. Los ensayos experimentales directamente sobre un prototipo del sistema suponen un elevado coste, pocos parámetros medibles y casi nula difusión de los resultados y comportamientos. La simulación numérica presenta un gran interés en cuanto a reducción de costes, aumento de parámetros medibles y número de variaciones en las distintas simulaciones.

Las normas internacionales de diseño (americanas y europeas), limitan la fuerza de parada (o de impacto) a valores comprendidos entre 4 y $8 \mathrm{kN}$ y limitan la elongación máxima a valores entre 1,1 y $1,75 \mathrm{~m}$ (Tabla 1 ).

\section{ESTUDIOS REALIZADOS}

Se exponen en este artículo los resultados de modelos numéricos que bajo distintas simulaciones nos permiten extraer conclusiones originales y cuantificar la influencia de valores propios de los materiales y del amortiguamiento del sistema. Los parámetros que se han considerado variables son la altura de caída $(h)$, la longitud de amarre $(L)$, la rigidez de la cuerda $(k)$, el amortiguamiento estructural $(\beta)$ y la masa del operario $(m)$.

Las distintas simulaciones realizadas permiten cuantificar la influencia de dichos parámetros y medir los valores resultantes correspondientes a aceleraciones y fuerzas máximas, alargamientos, factor de impacto y desarrollo temporal del mismo. Con todo ello se extraen conclusiones importantes sobre las configuraciones menos agresivas en los procesos de detención de la caída.

Se define el factor de impacto $(\gamma)$ como el cociente entre la máxima fuerza de retención sobre el accidentado y su peso

\section{FACTOR DE CAÍDA =}

Altura de caída

longitud de cuerda

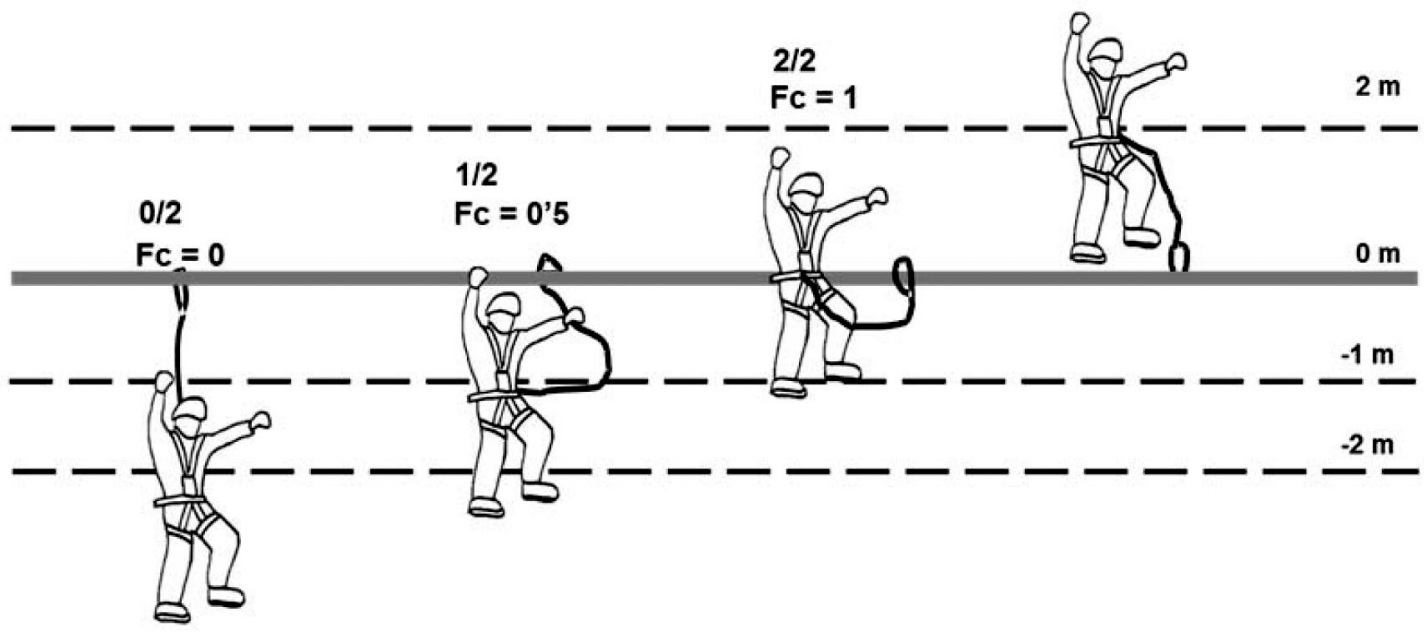

Figura 2. Factor de caída. 
estático. Admitiendo una aceleración máxima sensiblemente vertical, por tanto:

$$
\begin{gathered}
F-m g=m a \\
\gamma=\frac{F}{m g}=\frac{m(g+a)}{m g}=1+\frac{a}{g}
\end{gathered}
$$

Siendo:

$F$ la fuerza de impacto máxima $m$ la masa del lastre

$g$ la aceleración de la gravedad $a$ la aceleración máxima del lastre

Para el desarrollo del modelo numérico se han establecido las distintas geometrías que limitan la altura de caída y la longitud de cuerda que interviene en la absorción de energía.

La geometría inicial del equipo de amarre parte de su configuración estática bajo peso propio; esto es debido a la necesidad de cierta tensión y rigidez mínimas para la convergencia en los cálculos. La cuerda, bajo su peso propio y sujeta en dos puntos, adquiere forma de catenaria (Figura 3).

Las diversas configuraciones estudiadas consisten en simular una caída con equipos de amarre de 1 metro y de 2 metros de longitud de cuerda. Cada uno de estos equipos se ha modelado con una caída desde el punto más alto posible (factor de caída 2), y desde la misma horizontal que el punto de anclaje (factor de caída 1). Con lo que partimos de cuatro modelos geométricos distintos generados desde la ecuación general de la catenaria referida a unos ejes cualesquiera:

$$
Y+Y_{o}=a \operatorname{Ch} \frac{X-X_{o}}{a}
$$

El valor del parámetro $a$ se obtiene de la siguiente ecuación:

$$
\operatorname{Sh}\left[\frac{X_{1}(B)-X_{1}(A)}{2 a}\right]=\frac{\sqrt{L^{2}-\left[Y_{1}(B)-Y_{1}(A)\right]^{2}}}{2 a}
$$

Siendo $L$ la longitud del equipo de amarre.

Con lo que obtenemos cuatro modelos geométricos, donde el superíndice indica la altura de caída libre y el subíndice la longitud del equipo de amarre, así tenemos:

Mod $A_{1}^{2}$, con altura de caída $h=2 \mathrm{~m}$ y de longitud de equipo de amarre $L=1 \mathrm{~m}\left(F_{c}=2\right)$

Mod $B_{1}^{1}$, con altura de caída $h=1 \mathrm{~m}$ y de longitud de equipo de amarre $L=1 \mathrm{~m}\left(F_{c}=1\right)$

Mod $C_{2}^{4}$, con altura de caída $h=4 \mathrm{~m}$ y de longitud de equipo de amarre $L=2 \mathrm{~m}\left(F_{c}=2\right)$

Mod $D_{2}^{2}$, con altura de caída $h=2 \mathrm{~m}$ y de longitud de equipo de amarre $L=2 \mathrm{~m}\left(F_{c}=1\right)$

Por cuestiones de condicionamiento numérico de la convergencia del proceso, la puesta en carga bajo el peso propio se consigue introduciendo un pretensado de la cuerda (simulado mediante
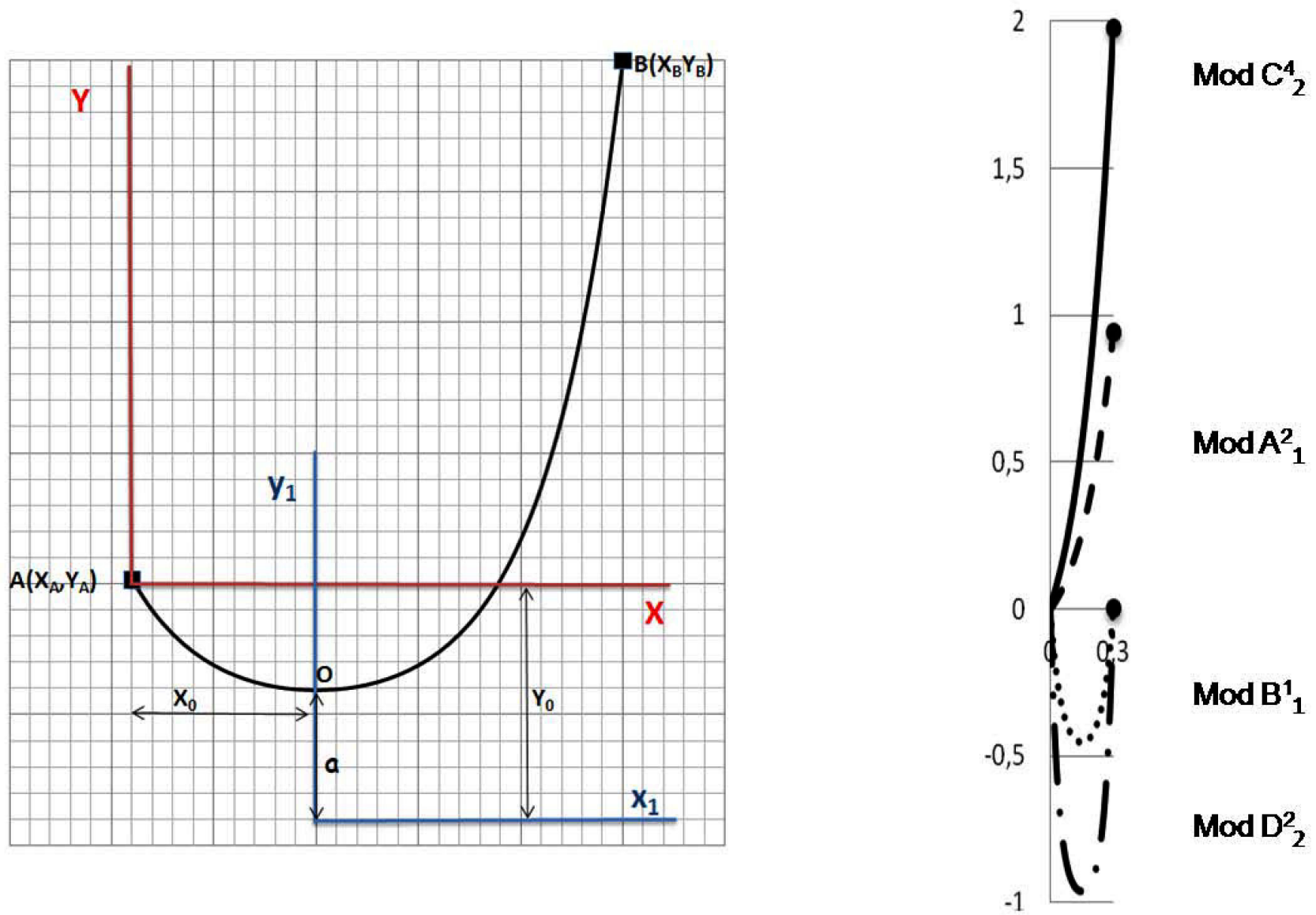

Figura 3. Geometría inicial del equipo de amarre. 
congelación) del mismo orden de magnitud que la solución final bajo el peso propio; este pretensado se elimina paulatinamente al mismo tiempo que se introduce la acción gravitatoria.

Para estas configuraciones se realizan las modelizaciones del sistema de protección, con el programa de elementos finitos Ansys (21), de un equipo de amarre (cuerda) con diámetro 10 $\mathrm{mm}$ y densidad $0,07 \mathrm{~kg} / \mathrm{m}$. Y un lastre de masa puntual de $100,1500200 \mathrm{~kg}$.

El fenómeno mecánico a modelar es muy complejo ya que presenta varios aspectos no lineales: se generan grandes movimientos, grandes giros e incluso grandes deformaciones (no linealidad geométrica). Se parte de una rigidez inicial transversal de la cuerda muy pequeña (la correspondiente al peso propio), que va aumentando conforme se tensa (rigidización por tensión).

Para tener en cuenta todas estas premisas, los elementos de Ansys utilizados han sido LINK10 para la cuerda y MASS21 para el lastre.

No se dispone en el momento de la redacción de este artículo de ensayos dinámicos de contraste del comportamiento del equipo de amarre. Aunque los autores realizaron ensayos cuasi estáticos en el laboratorio de elementos de seguridad de AIDICO en 2009 para determinar el comportamiento del material (cuerda de $\varnothing 10 \mathrm{~mm}$ y densidad $0,07 \mathrm{~kg} / \mathrm{m}$ ), su principal inconveniente para validar la ley de comportamiento es la velocidad de aplicación de la tracción, que influye notablemente y resulta muy inferior a la del impacto real.

Por todo ello se ha optado por utilizar una ley de comportamiento bilineal sin resistencia a la compresión y elástico lineal a tracción obtenida experimentalmente por Sulowski (6) con una rigidez en función del factor de caída. Por tanto, se ha considerado la rigidez $(k)$ de la cuerda ${ }^{2}$ de $42 \mathrm{kN}$ para un factor de caída 2 y de $33 \mathrm{kN}$ para un factor de caída 1 .

El multiplicador de la matriz de rigidez $(\beta)$ con el que se obtiene la matriz de amortiguamiento estructural ${ }^{3}$, se ha fijado analizando la repercusión de sus variaciones y la verosimilitud del fenómeno modelado mediante un análisis visual, quedando $\xi_{i}$ en un rango comprendido entre $10 \%$ y $30 \%$ del amortiguamiento crítico. Por tanto, $\beta$ será un parámetro a ajustar con mayor fiabilidad mediante experimentación en futuras investigaciones.

Para cada configuración en el modelo numérico se obtienen en función del tiempo:

- Los desplazamientos del lastre

- La aceleración del lastre, en particular su valor máximo

- Las reacciones en los soportes

- El factor de impacto

- La fuerza de parada, en particular su valor máximo

\section{RESULTADOS}

Los apartados siguientes recogen una selección de valores relevantes de las variables implicadas en el fenómeno para diversos valores de cada parámetro del modelo, manteniendo fijos los demás.

\subsection{En función de la altura de caída y factor de caída}

Fijados las rigideces, la masa y el amortiguamiento, se estudian los cuatro modelos geométricos definidos. Para estas simulaciones se toma un lastre de masa $100 \mathrm{~kg}$, una rigidez $k$ según (6) en función del factor de caída para una cuerda de nylon de $12 \mathrm{~mm}$ de diámetro $\left(k=42 \mathrm{kN}\right.$ para $F_{c}=2 \mathrm{y}$ $k=33 \mathrm{kN}$ para $\left.F_{c}=1\right)$; y un valor $\xi_{i}=0,1(10 \%$ del amortiguamiento crítico).

En la Tabla 2 se recogen los valores máximos de las principales variables implicadas.

Siendo para esta y las siguientes tablas:

$F_{c}$ el factor de caída

$U_{y}^{c}$ el desplazamiento vertical máximo del lastre (m)

$a_{y}$ la aceleración vertical máxima en el lastre en $\left(\mathrm{m} / \mathrm{s}^{2}\right)$

$a_{x}$ la aceleración horizontal máxima en el lastre $\left(\mathrm{m} / \mathrm{s}^{2}\right)$

$a_{\text {máx }}^{x}$ la resultante de la aceleración máxima

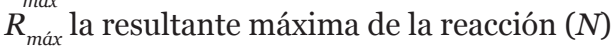

$\gamma$ el factor de impacto

$F_{y \text { máx }}$ la fuerza máxima de impacto vertical $(N)$

$E_{\text {máx }}$ la elongación máxima $(m)$

$t_{()}$el instante en que se alcanza los máximos (s)

Tabla 2. Resultados en función de la geometría (rigideces según $F_{c}$ ).

\begin{tabular}{|c|c|c|c|c|c|c|c|c|c|c|c|c|}
\hline & $F_{\mathrm{c}}$ & $\begin{array}{c}U_{Y} \\
(\mathbf{m})\end{array}$ & $\begin{array}{l}\boldsymbol{t}_{(U y)} \\
(\mathbf{s})\end{array}$ & $\begin{array}{c}a_{y} \\
\left(\mathrm{~m} / \mathrm{s}^{2}\right)\end{array}$ & $\begin{array}{l}t_{(a y)} \\
\text { (s) }\end{array}$ & $\begin{array}{c}a_{x} \\
\left(\mathrm{~m} / \mathrm{s}^{2}\right)\end{array}$ & $\begin{array}{l}t_{(a x)} \\
(\mathbf{s})\end{array}$ & $\begin{array}{c}a_{m a ́ x} \\
\left(\mathrm{~m} / \mathrm{s}^{2}\right)\end{array}$ & $\begin{array}{c}R_{\text {máx }} \\
(\mathrm{N})\end{array}$ & $Y$ & $\begin{array}{c}F_{y \text { máx }} \\
\text { (N) }\end{array}$ & $\begin{array}{l}E_{\text {máx }} \\
(\mathrm{m})\end{array}$ \\
\hline $\operatorname{Mod} A_{1}^{2}$ & 2 & $-2,12$ & 3,698 & 91,48 & 3,663 & $-24,98$ & 3,639 & 94,83 & 10.482 & 10,68 & 10.463 & 0,22 \\
\hline $\operatorname{Mod} B_{1}^{1}$ & 1 & $-1,14$ & 3,537 & 55,33 & 3,492 & $-15,99$ & 3,469 & 57,59 & 6.742 & 6,88 & 6.739 & 0,18 \\
\hline $\operatorname{Mod} C_{2}^{4}$ & 2 & $-4,44$ & 3,991 & 96,37 & 3,991 & 19,19 & 4,902 & 98,26 & 11.055 & 11,03 & 10.806 & 0,50 \\
\hline $\operatorname{Mod} D_{2}^{2}$ & 1 & $-2,37$ & 3,764 & 60,66 & 3,740 & 9,11 & 4,519 & 61,34 & 7.221 & 7,26 & 7.114 & 0,39 \\
\hline
\end{tabular}

${ }^{2} k$ : Pendiente de la recta que representa un comportamiento proporcional de la cuerda a tracción $(k=E A$; donde $E$ es el módulo elástico y $A$ el área de la sección de la cuerda).

${ }^{3}$ El índice de amortiguamiento modal $\xi_{i}=\pi \beta f_{i}$ se comporta linealmente con la frecuencia $f_{i}$ de un modo, siendo en el amortiguamiento de Rayleigh $[c]=\alpha[m]+\beta[k]$, con $\alpha=0$; este valor de $\xi_{i}$ se da para una frecuencia modal $f_{i}$, que en nuestro caso es del orden de $1 \mathrm{~Hz}$, por lo que: $\beta=\frac{\xi_{i}}{\pi}$ (expresando así más claramente la magnitud del amortiguamiento). 
La Figura 4 muestra las aceleraciones verticales obtenidas en el transcurso del proceso dinámico, durante $4 \mathrm{~s}$ para el equipo de amarre de 1 metro; y durante $7 \mathrm{~s}$ para el equipo de amarre de 2 metros. Se aprecia que se ha captado el proceso de detención de la caída, asegurando la obtención de las aceleraciones máximas.

Se observa claramente una diferencia significativa en función del factor de caída, y no de la altura de caída. Los mode$\operatorname{los} A_{1}^{2}$ y $C_{2}^{4}$ presentan aceleraciones máximas del orden de $10 \mathrm{~g}\left(100 \mathrm{~m} / \mathrm{s}^{2}\right)$, mientras que los Modelos $B_{1}^{1}$ y $D_{2}^{2}$ rondan los $6 \mathrm{~g}\left(60 \mathrm{~m} / \mathrm{s}^{2}\right)$, esto supone unas aceleraciones menores en un $40 \%$ en caídas con un factor 1 respecto a factores de caída 2.

El fenómeno presenta un efecto látigo de aceleración negativa en todas las geometrías estudiadas. El mínimo y máximo se alcanzan en instantes muy próximos (centésimas de segundo). Las caídas retenidas con un equipo de amarre de $1 \mathrm{~m}$. presentan dos picos de aceleración, mientras que las retenidas con el equipo de amarre de $2 \mathrm{~m}$. presentan tres. A partir de aquí en ambos casos el proceso dinámico se amortigua con un efecto pendular (con oscilaciones horizontales) hasta la detención.

El valor obtenido del desplazamiento máximo vertical proporciona la información necesaria para determinar la distancia libre de seguridad, en aras de evitar un impacto del lastre con el suelo u otro obstáculo. Esta variable, a diferencia de la aceleración, sí está influenciada por la altura de caída y por la longitud de cuerda, y no por el factor de caída (su cociente). Los modelos $A_{1}^{2}$ y $B_{1}^{1}$ con equipo de amarre de 1 metro experimentan una elongación aproximadamente tres veces menor que equipos de 2 metros.
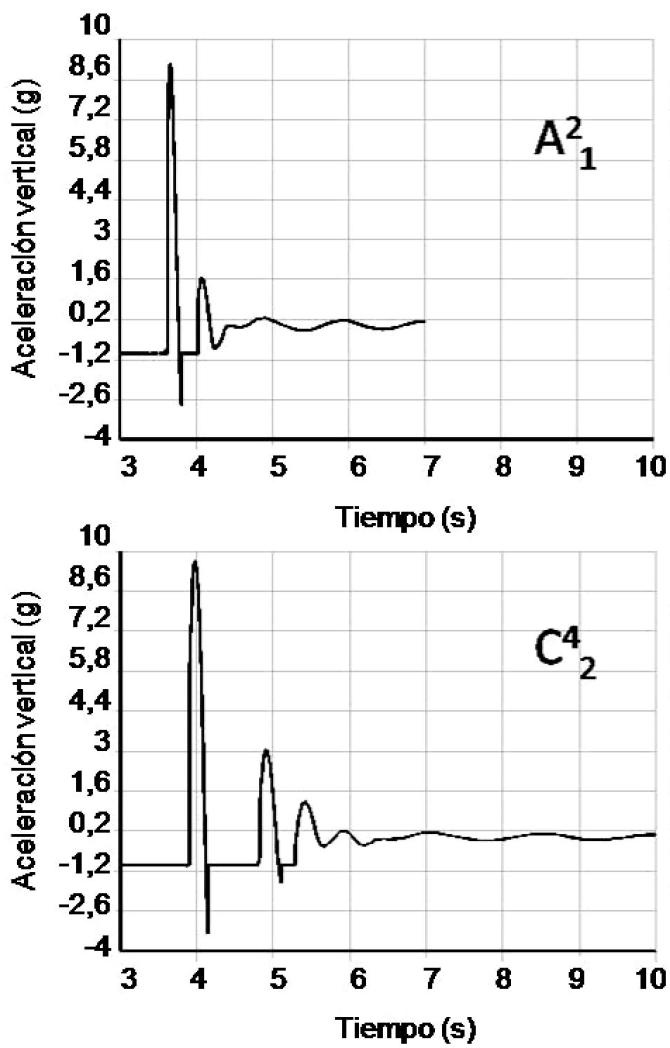

La aceleración horizontal tiene una magnitud considerablemente inferior a la vertical, ver Figura 5. Se alcanza el valor máximo para el modelo $A_{1}^{2}$. El primer valor extremo horizontal se alcanza centésimas de segundo antes del máximo vertical, (es el caso de los modelos $A_{1}^{2}$ y $B_{1}^{1}$ ) el siguiente valor se alcanza a más de un segundo (es el caso de los modelos $C_{2}^{4}$ y $D_{2}^{2}$ ). A mayor longitud del elemento de amarre, menor aceleración horizontal.

El factor de impacto $\gamma$ evidencia la gran transcendencia de la posición del anclaje respecto al lastre (usuario). El modelo $A^{2}$ sufre una fuerza de parada máxima un $35,5 \%$ mayor que el modelo $B_{1}^{1}$, ambos con un equipo de amarre de $1 \mathrm{~m}$. El modelo $C_{2}^{4}$ experimenta un incremento de un 33,6 \% en esta fuerza respecto al modelo $D_{2}^{2}$, ambos de $2 \mathrm{~m}$. La diferencia en la fuerzas de los modelos con factores de caída 2 es de 2,7 \%; para los modelos con factor de caída 1 es de $5,5 \%$. Se resalta que el factor de caída es un parámetro mucho más significativo en el fenómeno estudiado que la altura de caída.

En los cuatro modelos se presentan fuerzas de parada máximas superiores a los $6 \mathrm{kN}$ admitidos por las normas europeas de diseño.

Ahora bien, los modelos recogidos en la Tabla 2 presentan variaciones geométricas y de rigidez, lo que podría hacer pensar que las variaciones en los resultados son debidas al cambio de rigidez en el material (consecuencia de su distinto factor de caída). A continuación, en la Tabla 3 se recogen los resultados sin variación de la rigidez $(k=42 \mathrm{kN}$, independientemente de $F_{c}$ ) para los mismos modelos de la Tabla 2.

La variación de rigideces es de un $21 \%$, pero esto sólo modifica un $10 \%$, aproximadamente, la aceleración vertical sufrida
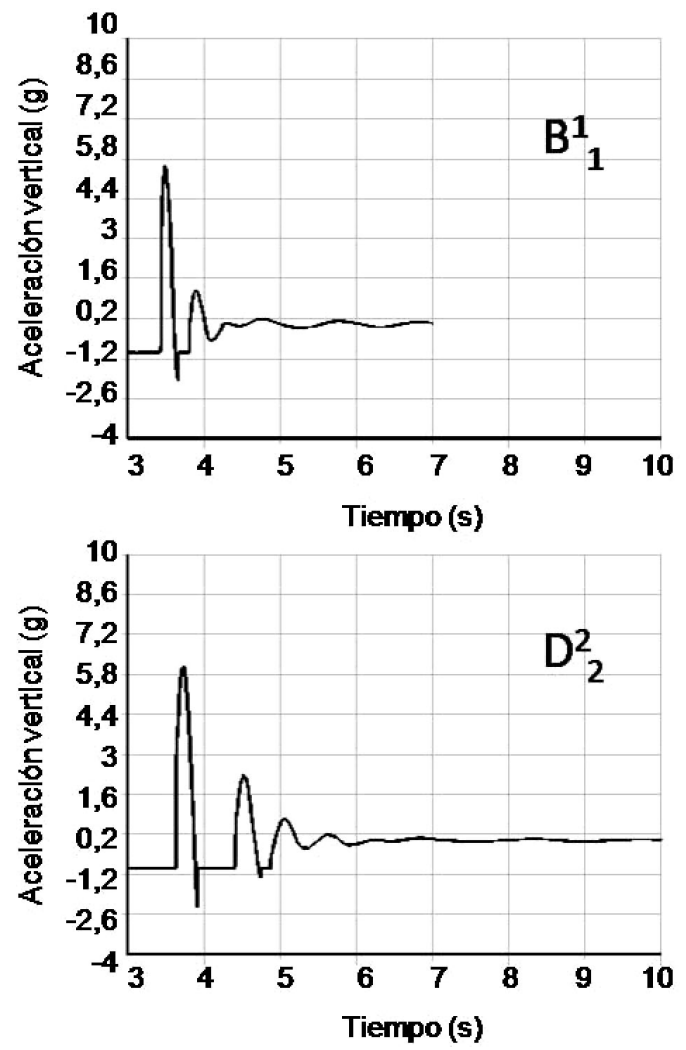

Figura 4. Aceleraciones verticales en los modelos analizados. 

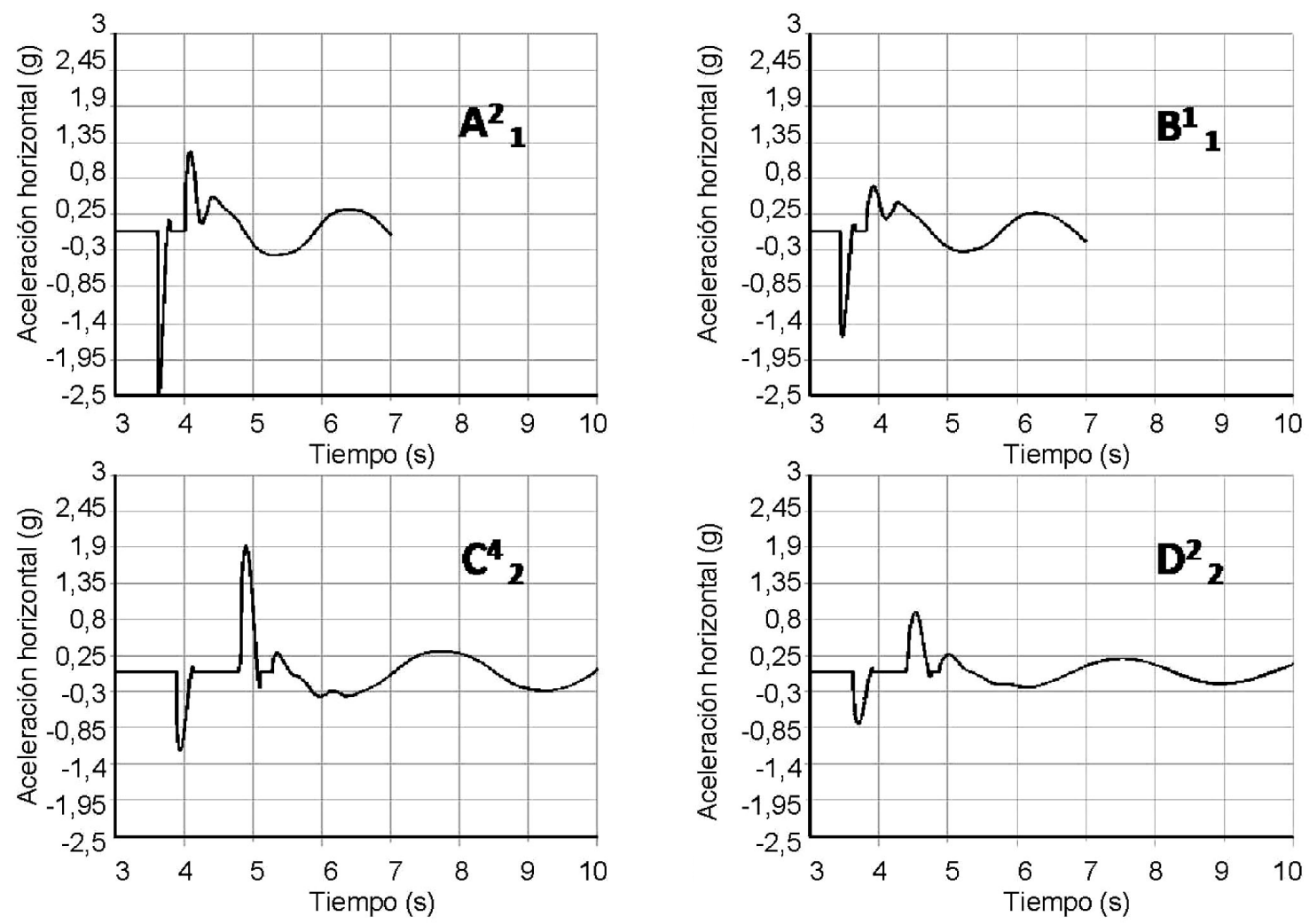

Figura 5. Aceleraciones horizontales en los modelos analizados.

por el lastre: comparando los modelos $B_{1}^{1}$ y $B_{1}^{\prime 1}$ esta variación supone $11 \%$, y un $9 \%$ en el caso de comparar los mode$\operatorname{los} D_{2}^{2}$ y $D_{2}^{\prime 2}$. En el resto de variables el comportamiento es similar (ver Tabla 3).

El modelo $A_{1}^{2}$ sufre una fuerza de parada máxima un 28,35\% mayor que el modelo $B_{1}^{\prime 1}$, ambos con un equipo de amarre de $1 \mathrm{~m}$. El modelo $C_{2}^{4}$ experimenta un incremento de esta fuerza de un 27,34 \% respecto al modelo $D_{2}^{\prime 2}$, ambos de $2 \mathrm{~m}$. La diferencia en la fuerzas de los modelos con factores de caída 2 es de 2,7\%; para los modelos con factor de caída 1 es de 4,5\%. Se resalta que el factor de caída es también un parámetro mucho más significativo que la rigidez en el fenómeno estudiado.

En definitiva, la Tabla 3 muestra la similitud en los comportamientos según el factor de caída. La variación de la rigidez de la cuerda en los modelos $B_{1}^{\prime 1}$ y $D_{2}^{\prime 2}$ (respecto a los modelos $B_{1}^{1}$ y $D_{2}^{2}$ ) ha supuesto un incremento de los valores próximo a un $5 \%$, lejos de la variación en los esfuerzos, que aumentan en un $30 \%$. Esto es fácilmente apreciable si comparamos los modelos $A_{1}^{2}$ con el $D_{2}^{2}$ o $D_{2}^{\prime 2}$ (en ambos casos la altura de caída es de $2 \mathrm{~m}$ ).

\subsection{En función de la rigidez del equipo de amarre (k)}

Se exponen los resultados para el modelo $C_{2}^{4}$ (Figura 3), que corresponde a un factor de caída 2 y una longitud de $2 \mathrm{~m}$ de equipo de amarre. Este modelo es el que presenta los valores más perjudiciales para el accidentado entre los cuatro estudiados. Para estas simulaciones se toma un lastre de masa $100 \mathrm{~kg}$ y un valor $\xi_{i}=0,1$ (10 \% del amortiguamiento crítico).

En la Tabla 4 se recogen los valores máximos de las principales variables implicadas.

La rigidez $k$ de la referencia de Sulowski (6) es de $42 \mathrm{kN}$ para $F_{c}=2$ y cuerda de $12 \mathrm{~mm}$. Analizando las rigideces en un rango comprendido entre 30 y $50 \mathrm{kN}$ se abarcan ya (para este $F_{c}$ ) diámetros sensiblemente inferiores y superiores a los estudiados por dicho autor. Este rango puede incluir también variaciones de materiales en la composición del equipo de amarre, dado que el ensayo empírico de (6) sólo contemplaba tres tipos de material. Pero las simulaciones numéricas nos permiten con facilidad analizar casos mucho más radicales como los de las rigideces de 1 y $100 \mathrm{kN}$.

Tabla 3. Resultados en función de la geometría (con igual rigidez).

\begin{tabular}{|c|c|c|c|c|c|c|c|c|c|c|c|c|}
\hline & $F_{\mathrm{c}}$ & $\begin{array}{c}U_{Y} \\
(\mathbf{m})\end{array}$ & $\begin{array}{l}t_{(U y)} \\
(\mathbf{s})\end{array}$ & $\begin{array}{c}a_{y} \\
\left(\mathrm{~m} / \mathbf{s}^{2}\right)\end{array}$ & $\begin{array}{l}t_{(a y)} \\
\text { (s) }\end{array}$ & $\begin{array}{c}a_{x} \\
\left(\mathrm{~m} / \mathbf{s}^{2}\right)\end{array}$ & $\begin{array}{l}t_{(a x)} \\
(\mathbf{s})\end{array}$ & $\begin{array}{c}a_{m a ́ x} \\
\left(\mathrm{~m} / \mathrm{s}^{2}\right)\end{array}$ & $\begin{array}{l}R_{\text {máx }} \\
\text { (N) }\end{array}$ & $\mathbf{Y}$ & $\begin{array}{c}F_{y \text { máx }} \\
(\mathbf{N})\end{array}$ & $\begin{array}{l}E_{\text {máx }} \\
\text { (m) }\end{array}$ \\
\hline $\operatorname{Mod} A_{1}^{2}$ & 2 & $-2,12$ & 3,698 & 91,48 & 3,663 & $-24,98$ & 3,639 & 94,83 & 10.482 & 10,68 & 10.463 & 0,22 \\
\hline Mod $B_{1}^{\prime 1}$ & 1 & $-1,12$ & 3,514 & 62,36 & 3,476 & $-18,91$ & 3,462 & 65,16 & $7 \cdot 517$ & 7,65 & 7.496 & 0,16 \\
\hline $\operatorname{Mod} C_{2}^{4}$ & 2 & $-4,44$ & 3,991 & 96,37 & 3,991 & 19,19 & 4,902 & 98,26 & 11.055 & 11,03 & 10.806 & 0,50 \\
\hline $\operatorname{Mod} D_{2}^{\prime 2}$ & 1 & $-2,30$ & 3,747 & 68,11 & 3,717 & $-9,08$ & 3,697 & 68,71 & 7.918 & 8,01 & 7.851 & 0,32 \\
\hline
\end{tabular}


Tabla 4. Resultados en función de la rigidez del equipo de amarre.

\begin{tabular}{|c|c|c|c|c|c|c|c|c|c|c|c|}
\hline $\begin{array}{c}\boldsymbol{k} \\
(\mathbf{k N})\end{array}$ & $\begin{array}{c}\boldsymbol{U}_{\mathbf{Y}} \\
(\mathbf{m})\end{array}$ & $\begin{array}{c}\boldsymbol{t}_{(U y)} \\
(\mathbf{s})\end{array}$ & $\begin{array}{c}\boldsymbol{a}_{y} \\
\left(\mathbf{m} / \mathbf{s}^{\mathbf{2}}\right)\end{array}$ & $\begin{array}{c}\boldsymbol{t}_{(a y)} \\
(\mathbf{s})\end{array}$ & $\begin{array}{c}\boldsymbol{a}_{\boldsymbol{x}} \\
\left(\mathbf{m} / \mathbf{s}^{\mathbf{2}}\right)\end{array}$ & $\begin{array}{c}\boldsymbol{t}_{(a x)} \\
(\mathbf{s})\end{array}$ & $\begin{array}{c}\boldsymbol{a}_{\text {máx }} \\
\left(\mathbf{m} / \mathbf{s}^{\mathbf{2}}\right)\end{array}$ & $\begin{array}{c}\boldsymbol{R}_{\text {máx }} \\
(\mathbf{N})\end{array}$ & $\mathbf{\gamma}$ & $\begin{array}{c}\boldsymbol{F}_{y \text { máx }} \\
(\mathbf{N})\end{array}$ & $\begin{array}{c}\boldsymbol{E}_{\text {máx }} \\
(\mathbf{m})\end{array}$ \\
\hline 1 & $-10,00$ & 4,776 & 19,88 & 4,776 & 0,95 & 7,675 & 19,90 & 3.007 & 3,03 & 2.970 & 6,03 \\
\hline 10 & $-5,20$ & 4,138 & 51,63 & 4,138 & 15,25 & 5,655 & 53,83 & 6.406 & 6,49 & 6.363 & 1,23 \\
\hline 30 & $-4,57$ & 4,023 & 83,26 & 3,994 & 19,62 & 5,065 & 85,54 & 9.692 & 9,73 & 9.534 & 0,60 \\
\hline 50 & $-4,39$ & 3,982 & 106,14 & 3,963 & 18,29 & 4,825 & 107,71 & 11.859 & 11,99 & 11.751 & 0,43 \\
\hline 100 & $-4,23$ & 3,965 & 155,74 & 3,924 & $-21,98$ & 3,914 & 157,29 & 16.822 & 17,05 & 16.709 & 0,27 \\
\hline
\end{tabular}

El desplazamiento máximo vertical del lastre para una rigidez de $k=1 \mathrm{kN}$ resulta excesivo en la mayoría de las construcciones habituales. Estos sistemas deben evitar que el operario durante la caída alcance una superficie inferior, en raras ocasiones se dispone de una distancia de seguridad tan amplia bajo el operario. Un equipo así diseñado tendría muy poca versatilidad.

Un equipo con una rigidez tan grande como $k=100 \mathrm{kN}$ proporciona una detención con muy poco desplazamiento, pero dispara las aceleraciones y la fuerza máxima de parada a valores intolerables para el cuerpo humano, rozando los $16 \mathrm{~g}$ y superando $16 \mathrm{kN}$, respectivamente.

Obtener un equilibrio entre ambas variables (desplazamiento y fuerza máxima de parada) es primordial en estos sistemas de protección individual, así como en otros dispositivos de seguridad con absorción de energía. Considerando únicamente la rigidez del material, sin atender a otro tipo de factores como son la durabilidad, este equilibrio estaría en materiales con una rigidez $k$ de unos $10 \mathrm{kN}$ (en equipos sin absorbedor de energía).

\subsection{En función de la masa del operario}

Fijados las rigideces y el amortiguamiento, se estudian los cuatro modelos geométricos definidos en la Figura 3. Para estas simulaciones se vuelve a tomar la rigidez $k$ en función del factor de caída para una cuerda de nylon de $12 \mathrm{~mm}$ de diámetro, según (6). Se toma un valor $\xi_{i}=0,1$ (10 \% del amortiguamiento crítico).

La Tabla 5 muestra los valores de las variables más significativas para cada modelo estudiado en función de la masa del lastre.

La variación en el desplazamiento del lastre no resulta ser excesivamente significativa; el peor de los casos no alcanza los o,30 m (Modelo $C_{2}^{4}$ ). Se aprecia un comportamiento lineal, muy interesante para proceder a interpolaciones para distintas masas.

Las aceleraciones máximas sufridas por el lastre y el factor de impacto van disminuyendo y, por el contrario, aumentando la fuerza máxima de parada. Por tanto, la variable significativa a la hora de determinar las posibles lesiones en el operario retenido durante su caída debe ser la fuerza máxima de parada.

En referencia a la fuerza máxima de parada sufrida por el lastre, se aprecia un incremento sustancial que varía entre el 47 y $57 \%$, según modelo, al variar el lastre de 100 a $200 \mathrm{~kg}$. En todos los modelos se superan los $6 \mathrm{kN}$ fijados por la nor-

Tabla 5. Resultados en función de la masa del operario.

\begin{tabular}{|c|c|c|c|c|c|c|c|c|c|c|c|c|}
\hline & $\begin{array}{c}\text { Masa } \\
(\mathrm{kg})\end{array}$ & $\begin{array}{c}U_{Y} \\
(\mathbf{m})\end{array}$ & $\begin{array}{l}t_{(U y)} \\
(\mathbf{s})\end{array}$ & $\begin{array}{c}a_{y} \\
\left(\mathrm{~m} / \mathbf{s}^{2}\right)\end{array}$ & $\begin{array}{l}t_{(a y)} \\
\text { (s) }\end{array}$ & $\begin{array}{c}a_{x} \\
\left(\mathrm{~m} / \mathrm{s}^{2}\right)\end{array}$ & $\begin{array}{l}t_{(a x)} \\
(\mathbf{s})\end{array}$ & $\begin{array}{c}a_{\text {máx }} \\
\left(\mathrm{m} / \mathrm{s}^{2}\right)\end{array}$ & $\begin{array}{c}R_{\text {máx }} \\
(\mathrm{N})\end{array}$ & $\gamma$ & $\begin{array}{c}F_{y \text { máx }} \\
(\mathrm{N})\end{array}$ & $\begin{array}{l}E_{\text {máx }} \\
(\mathbf{m})\end{array}$ \\
\hline $\operatorname{Mod} A_{1}^{2}$ & 100 & $-2,118$ & 3,698 & 91,48 & 3,663 & $-24,98$ & 3,639 & 94,83 & 10.482 & 10,68 & 10.463 & 0,22 \\
\hline $\operatorname{Mod} A_{1}^{2}$ & 150 & $-2,184$ & 3,702 & 74,21 & 3,682 & $-18,47$ & 3,651 & 76,47 & 12.971 & 8,80 & 12.941 & 0,28 \\
\hline $\operatorname{Mod} A_{1}^{2}$ & 200 & $-2,253$ & 3,734 & 65,09 & 3,706 & 16,28 & 4,298 & 67,09 & 15.436 & 7,85 & $15 \cdot 379$ & 0,35 \\
\hline Mod $B_{1}^{1}$ & 100 & $-1,144$ & 3,537 & 55,33 & 3,492 & $-15,99$ & 3,469 & 57,59 & 6.742 & 6,88 & 6.739 & 0,18 \\
\hline Mod $B_{1}^{1}$ & 150 & $-1,208$ & 3,542 & 45,68 & 3,517 & $-12,31$ & 3,490 & 47,31 & 8.602 & 5,83 & 8.567 & 0,24 \\
\hline Mod $B_{1}^{1}$ & 200 & $-1,268$ & 3,570 & 40,18 & 3,544 & $-10,27$ & 3,500 & 41,47 & 10.299 & 5,23 & 10.254 & 0,33 \\
\hline $\operatorname{Mod} C_{2}^{4}$ & 100 & $-4,442$ & 3,991 & 96,37 & 3,991 & 19,19 & 4,902 & 98,26 & 11.055 & 11,03 & 10.806 & 0,60 \\
\hline $\operatorname{Mod} C_{2}^{4}$ & 150 & $-4,589$ & 4,014 & 80,87 & 4,014 & 20,03 & 5,095 & 83,31 & 14.020 & 9,50 & 13.967 & 0,63 \\
\hline $\operatorname{Mod} C_{2}^{4}$ & 200 & $-4,724$ & 4,067 & 70,47 & 4,026 & 19,36 & 5,251 & 73,08 & 16.918 & 8,46 & 16.576 & 0,76 \\
\hline $\operatorname{Mod} D_{2}^{2}$ & 100 & $-2,366$ & 3,764 & 60,66 & 3,740 & 9,11 & 4,519 & 61,34 & 7.221 & 7,26 & 7.114 & 0,39 \\
\hline $\operatorname{Mod} D_{2}^{2}$ & 150 & $-2,501$ & 3,788 & 50,13 & 3,753 & 10,03 & 4,710 & 51,13 & 9.357 & 6,22 & 9.139 & 0,52 \\
\hline $\operatorname{Mod} D_{2}^{2}$ & 200 & $-2,611$ & 3,830 & 45,00 & 3,792 & 10,11 & 4,838 & 46,12 & 11.229 & 5,71 & 11.184 & 0,63 \\
\hline
\end{tabular}


ma UNE-EN 355. Al aumentar el peso del lastre, los valores de las fuerzas máximas de parada alcanzan valores muy alejados de la seguridad. Por tanto, la norma debería exigir la especificación por parte de los fabricantes del peso máximo del usuario que mantiene el valor de la fuerza máxima de parada dentro de los límites seguros para la integridad del mismo.

\subsection{En función del amortiguamiento}

El efecto de esta variable se expone en la Tabla 6 para el modelo $C_{2}^{4}$, que corresponde a un factor de caída 2 y una longitud de $2 \mathrm{~m}$ de equipo de amarre. Este modelo es el que presenta, tal y como ya se ha comentado, los valores más perjudiciales para el accidentado entre los cuatro modelos analizados.

Para estas simulaciones se toma un lastre de masa $100 \mathrm{~kg}$ y una rigidez de $k=42 \mathrm{kN}$, tomada de (6) para una cuerda de nylon de $12 \mathrm{~mm}$ de diámetro.

Se presentan los resultados para las simulaciones con valores de $\xi_{i}=$ 0,1, 0,2 y 0,3 veces el amortiguamiento crítico. Este será un parámetro a ajustar con mayor fiabilidad mediante experimentación en futuras investigaciones, dado que en el momento de redacción de este artículo no se dispone de medios para acotar más este rango.

Se aprecia un claro aumento no lineal del factor de impacto con el índice de amortiguamiento. La aceleración máxima y la fuerza de parada máxima aumentan al estar el sistema más amortiguado.

\section{CONCLUSIONES}

Con un mismo factor de caída, la altura de la misma no resulta decisiva en cuanto a la aceleración sufrida por el lastre (Tabla 2). Duplicando la altura de caída no se presenta un aumento mayor del $10 \%$ en la aceleración sufrida por el lastre. Por tanto, la altura de caída no es un factor determinante en cuanto a la severidad se refiere.

El factor de caída influye notablemente en las aceleraciones que sufre el lastre. Los modelos con factor de caída 2 presentan, en promedio, aceleraciones del orden de $10 \mathrm{~g}$; y los de factor 1 , de $6 \mathrm{~g}$ (en estas simulaciones se ha variado también la rigidez). Esto supone una reducción de un $40 \%$ en aceleración a sufrir por el accidentado (Tabla 2). Por lo tanto, el factor de caída (determinado por la ubicación del punto de anclaje respecto del usuario) es de vital importancia. Si se estudia el fenómeno sin variar rigideces, esta reducción en la aceleración baja a un 30 \%, refrendándose la afirmación anterior: el factor de caída resulta ser determinante.

Independientemente del modelo geométrico estudiado, siempre predomina la componente vertical de la aceleración. No obstante, en el modelo $C_{2}^{4}$ podemos apreciar que la componente horizontal puede llegar a alcanzar valores del $20 \%$ de la componente vertical.

En cuanto la rigidez del sistema (Tabla 4), el uso de un equipo muy poco rígido transmite muy poca aceleración al accidentado, lo cual resultaría favorable; pero, en contrapartida, la excesiva deformación hace que estos equipos sean inoperantes. Un equipo muy rígido transmite unas aceleraciones intolerables por el cuerpo humano.

La masa del operario (Tabla 5) influye notablemente en la fuerza máxima de parada que recibe el usuario. Variando la masa de $100 \mathrm{~kg}$ a $200 \mathrm{~kg}$, la fuerza de impacto aumenta del orden de un $50 \%$, a pesar de que las aceleraciones máximas disminuyan.

El amortiguamiento (Tabla 6) es un parámetro dado por el sistema. No obstante, gracias a las posibilidades de los métodos numéricos, se puede estudiar el comportamiento variando este parámetro y así conseguir datos válidos para determinar cuál sería el amortiguamiento óptimo del sistema. Los resultados de los modelos indican mayores impactos para sistemas con mayor amortiguamiento estructural.

Finalmente, a la vista de los resultados, el sistema compuesto únicamente por el equipo de amarre con las cuerdas habituales (poliamida de alta tenacidad, diámetro 10-15 mm, unos $40 \mathrm{kN}$ de rigidez) supera los límites establecidos por las normas europeas, llegando a duplicar la fuerza máxima de parada para un factor de caída de 2 y comportamiento elástico. Según el estudio de rigideces realizado, para no superar dichos límites, la cuerda debería tener una rigidez del orden de la cuarta parte (la cuarta parte de sección, con el mismo módulo elástico); pero ello aumentaría la elongación a valores superiores a $1 \mathrm{~m}$, rondando los valores mínimos establecidos, y sobrepasaría el rango elástico, poniendo en entredicho su propia resistencia. Por tanto, con estas cuerdas se hace preciso contar con la deformabilidad de otros elementos del sistema como el propio arnés o el absorbedor de energía (fusible) que incorporan algunos equipos; en otro caso los impactos serían inadmisibles. Desde el punto de vista de los modelos numéricos, se concluye la necesidad de incorporar el comportamiento elastoplástico en la propia cuerda y en los elementos adicionales (arnés y/o absorbedor) que deberían incluirse en futuras investigaciones.

Tabla 6. Resultados en función del amortiguamiento.

\begin{tabular}{|c|c|c|c|c|c|c|c|c|c|c|c|c|}
\hline & $\xi_{i}$ & $\begin{array}{c}U_{Y} \\
(\mathbf{m})\end{array}$ & $\begin{array}{l}t_{(U y)} \\
(\mathbf{s})\end{array}$ & $\begin{array}{c}a_{y} \\
\left(\mathbf{m} / \mathbf{s}^{2}\right)\end{array}$ & $\begin{array}{l}t_{(a y)} \\
(\mathbf{s})\end{array}$ & $\begin{array}{c}a_{x} \\
\left(\mathbf{m} / \mathbf{s}^{2}\right)\end{array}$ & $\begin{array}{l}t_{(a x)} \\
(\mathrm{s})\end{array}$ & $\begin{array}{c}a_{m a ́ x} \\
\left(\mathbf{m} / \mathbf{s}^{2}\right)\end{array}$ & $\begin{array}{l}R_{\text {máx }} \\
(\mathrm{N})\end{array}$ & $\gamma$ & $\begin{array}{c}F_{y \text { máx }} \\
(\mathbf{N})\end{array}$ & $\begin{array}{l}E_{\text {máx }} \\
\text { (m) }\end{array}$ \\
\hline $\operatorname{Mod} C_{2}^{4}$ & 0,1 & $-4,44$ & 3,991 & 96,37 & 3,991 & 19,19 & 4,902 & 98,26 & 11.055 & 11,03 & 10.806 & 0,50 \\
\hline Mod $C_{2}^{4}$ & 0,2 & $-4,34$ & 3,995 & 108,41 & 3,918 & $-16,73$ & 3,906 & 109,69 & 11.992 & 12,19 & 11.949 & 0,38 \\
\hline $\operatorname{Mod} C_{2}^{4}$ & 0,3 & $-4,28$ & 3,988 & 155,02 & 3,906 & $-22,62$ & 3,906 & 156,66 & 16.650 & 16,99 & 16.646 & 0,32 \\
\hline
\end{tabular}




\section{REFERENCIAS}

(1) AENOR. (2009). UNE-EN 363:2009. Equipos de protección individual contra caídas. Sistemas de protección individual contra caídas. Madrid: Asociación Española de Normalización.

(2) Sulowski, A.C. (2006). How good is the $8 \mathrm{kN}$ Maximum Arrest Force limit in Industrial Fall Arrest Systems? Seattle: WA: International Society for Fall Protection.

(3) Wang, C.H. (1977). Free Fall Restraint Systems. Professional Safety - ASSE Journal, 22(2).

(4) Steinberg, H.L. (1977). NBSIR 76-1146 A Study of Personal Fall-Safety Equipment. Washington, D.C.: National Bureau of Standards.

(5) Microys, H.F. (1977). Climbing Ropes. American Alpine Journal, 21(1): 130-147.

(6) Sulowski, A.C., et al. (1981). Assessing Fall Arresting System Effectiveness. Parts 1 and 2. National Safety News, 123(3-4).

(7) Brinkley, J. W., Sulowski, A.C. (1990). Measurement of Maximum Arrest Force in Performance Test of Fall Protection Equipment. Journal of Testing and Evaluation, 18(2): 123-127, doi: http://dx.doi.org/10.1520/JTE12462J.

(8) Irles Más, R., González Sánchez, A., Segovia Eulogio, E., Maciá Mateu, A. (2002). Las redes verticales de seguridad en la construcción de edificios I. Informes de la Construcción, 53(477): 21-29, doi: http://dx.doi.org/10.3989/ic.2002.v53. i477.639.

(9) Segovia Eulogio, E., Irles Más, R., González Sánchez, A., Maciá Mateu, A., Pomares Torres, J.C. (2007). Las redes verticales de seguridad en la construcción de edificios II. Informes de la Construcción, 59(505): 37-51, doi: http://dx.doi. org/10.3989/ic.2007.v59.i505.499.

(10) González, M.N., Cobo, A., Fuente, J. V., Bresó, S., Lozano, C. (2007). Comportamiento bajo cargas estáticas de sistemas provisionales de protección de borde realizados con elementos de acero. Informes de la Construcción, 63(521): 57-67, doi: http://dx.doi.org/10.3989/ic.09.070.

(11) González, M.N., Cobo, A., Fuente, J. V. (2013). Obtención de modelos de cálculo de sistemas provisionales de protección de borde mediante la técnica de Análisis Modal Operacional. Informes de la Construcción, 65(529): 99-106, doi: http:// dx.doi.org/10.3989/ic.11.133.

(12) Cobo, A., Bastidas, D. M., González, M. N., Medina, E., Bastidas, J. M. (2011). Ductility in a new low nickel stainless steel for reinforced concrete. Materiales de Construcción, 61(304): 613-620, doi: http://dx.doi.org/10.3989/mc.2011.57210.

(13) Pomares, J.C., Irles, R., Segovia, E.C., Boixader, D. (2013). Barandillas de protección personal con solicitación dinámica. Informes de la Construcción, 65(530): 241-251, doi: http://dx.doi.org/ 10.3989/ic.11.040.

(14) Comunidad Europea. (1989, 30 de diciembre). Directiva 89/686/CEE, de 21 de diciembre de 1989, sobre aproximación de las legislaciones de los estados miembros relativas a los equipos de protección individual. Diario Oficial de la Unión Europea, ${ }^{0}$ 399, pp. 18-38.

(15) AENOR. (1993). UNE-EN 364:1993. Equipos de protección individual contra caídas de altura. Métodos de ensayo. Madrid: Asociación Española de Normalización.

(16) AENOR. (2002). UNE-EN 355:2002. Equipos de protección individual contra caídas de altura. Absorbedores de energía. Madrid: Asociación Española de Normalización.

(17) ASSE. (2004). ANSI A10.32-2004. Fall Protection Systems-American National Standard for Construction and Demolition Operations. Illinois: American Society of Safety Engineers.

(18) ANSI/ASSE. (2007). Z359.1-20o7 Safety Requirements for Personal Fall Arrest. Systems, Subsystems and Components. Illinois: American Society of Safety Engineers.

(19) ANSI/ASSE. (2009). Z359.13-2009 Personal Energy Absorbers and Energy Absorbing Lanyards. Illinois: American Society of Safety Engineers.

(20) ISO. (2000). ISO 10333-2:200o. Personal fall-arrest systems - Part 2: Lanyards and energy absorbers. International Organization for Standardization.

(21) Ansys Inc. (2003). ANSYS 8.1. http://www.ansys.com/. 